\title{
miR-543 inhibits tumor malignant progression by regulating TWIST1 in human gastric cancer
}

Guoda Lian

Sun Yat-Sen University

Xuanna Li

Sun Yat-Sen University

Shaojie Chen

Sun Yat-Sen University

Jiajia Li

Sun Yat-Sen University

Yaqing Li

Sun Yat-Sen University

Shangxiang Chen

Sun Yat-Sen University

Xiangan Lin

Sun Yat-Sen University

Haoming Lin

Sun Yat-Sen University

Kaihong Huang ( $\square$ huangkh@mail.sysu.edu.cn )

Sun Yat-Sen University

Yinting Chen

Sun Yat-Sen University

\section{Research}

Keywords: Gastric cancer, TWIST1, miR-543

Posted Date: February 13th, 2020

DOI: https://doi.org/10.21203/rs.2.23437/v1

License: (1) This work is licensed under a Creative Commons Attribution 4.0 International License. Read Full License 


\section{Abstract}

Background: TWIST1, a highly conserved basic helix-loop-helix (bHLH) transcription factor, is essential to epithelial-mesenchymal transition (EMT) and cancer metastasis in gastric cancer (GC). However, little is known about the post-transcriptional regulation of TWIST1, especially the mechanisms involving microRNAs.

Methods: TWIST1 expression were observed on 107 cases of gastric cancer through tissue microarray technology to identify their correlations with clinicopathological parameters and patient survival. The regulation of TWIST1 by miR-543 was confirmed by western blot, dual luciferase activity assays and rescue experiments. Moreover, the functions of miR-543 on cell migration, invasion, tumorigenicity and metastatic potential were evaluated by stably expression strategy in vitro and in vivo.

Results: TWIST1 is overexpressed in 74 of 107 GC tumor samples. High TWIST1 expression positively correlated with poor prognosis of GC patients. In addition, TWIST1 was found to be a direct target of miR543. Expression of miR-543 was obviously decreased in GC cell lines and primary tissues. Overexpression of miR-543 in GC cells inhibited proliferation, colony formation, migration and invasion by suppressing expression of TWIST1. Ectopic expression of miR-543 inhibited tumor growth and prevented liver metastasis of gastric cancer cells in mice.

Conclusion: TWIST1 is overexpressed in gastric cancer and regulated by miR- 543 . Downregulated miR543 promotes tumor growth and metastasis of GC, indicating the possibility of new strategies for GC therapy.

\section{Background}

Gastric cancer is one of the most frequently diagnosed and aggressive carcinomas in the world [1]. It is highly prevalent among Chinese and certain East Asian populations [2]. Although gastric cancer is considered surgical and chemosensitivity, the prognosis is still poor mainly because of local invasion and tumor metastasis, and an overall 5 -year survival rate is about $20 \%$ in most areas of the world $[3,4]$.

Epithelial-mesenchymal transition (EMT) is known to be a central mechanism responsible for invasiveness and metastasis in diverse solid tumors, including gastric cancer $[5,6]$. TWIST1, a highly conserved basic helix-loop-helix (bHLH) transcription factor, has been demonstrated to play an important role in the promotion of EMT and cancer metastasis [7]. One major mechanism of TWIST1 inducing EMT is through binding to its E-box consensus sites in gene promoters, ultimately leading to transcriptional activation or repression $[8,9]$. Meanwhile, TWIST1 has also been suggested to have oncogenic properties. Ansieau et al. found that overexpression of TWIST1 inhibits oncogene-induced senescence and apoptosis and interferes with both p53- and RB- dependent pathways [10]. Using transgenic mouse models, Morel et al. found that TWIST1 fosters cell malignant transformation and primary tumor growth [11]. TWIST1 also promotes gastric cancer proliferation through modulating cell cycle progression [12]. 
Besides, numerous studies have shown that TWIST1 protein is upregulated in various cancer types and correlates with poor clinical prognosis [13-15].

Several studies indicated that TWIST1 expression is transcriptionally regulated by EGFR/STAT3 and NFKB signaling $[16,17]$. Accumulating evidence also indicates TWIST1 could be posttranslational modification by MAPKs or AKT1 in breast cancer $[18,19]$. Moreover, epigenetic modification is another important mechanism that regulates the expression of TWIST1. MicroRNAs (miRNAs) are short, 20- to 22nucleotides RNA molecules that negatively regulate gene expression by repressing mRNA translation or cleaving target of mRNAs $[20,21]$. Studies also indicated that TWIST1 is subject to posttranscriptional regulation by microRNAs $[22,23]$. However, the mechanism and role of elevated TWIST1 expression in GC remains largely unknown.

In this study, we found that TWIST1 expression is significantly upregulated in GC. We also found miR-543 directly target TWIST1. And miR-543 is downregulated in human gastric cancer cells and clinical samples. The antiproliferative and antimetastatic properties of miR-543 through suppressing TWIST1 expression were verified by a subset of in vitro and in vivo assays.

\section{Methods}

Human tissue samples and cell lines

Pair-matched tumorous and adjacent nontumorous gastric tissues from 33 patients undergoing resection for gastric cancer were obtained from Sun Yat-Sen Memorial Hospital of Sun Yat-Sen University (Guangzhou, China). Tissue microarray (TMA) containing 107 gastric cancer tumor tissue constructed as described in our previous study [24]. The study was carried out in accordance with the Declaration of Helsinki (2000). Prior patients' consent and approval from the Research Ethics Committee of Sun Yat-Sen Memorial Hospital were obtained. Human gastric carcinoma cell lines AGS, SGC7901, HGC-27 and BGC823 were obtained from Institute of Biochemistry and Cell Biology, Chinese Academy of Sciences (Shanghai, China). Non-malignant human gastric epithelial immortalized cell line GES-1 was purchased from Beijing Institute for Cancer Research Collection. All cell lines were cultured in DMEM supplemented with $10 \%$ fetal bovine serum (Gibco).

RNA Isolation And Quantitative RT-PCR

Total RNA from tissue samples and cell lines was extracted using TRIzol (Invitrogen) following the manufacturer's protocol. cDNA was synthesized from $1 \mu \mathrm{g}$ of total RNA using the PrimerScript RT-PCR kit (Takara Bio, Otsu, Japan). Mature miRNAs were reverse transcribed using the M-MLV reverse transcriptase (Promega). The mature miRNA sequence-specific stem-loop primers were: miR-543, 5'GTCGTATCCAGTGCAGGGTCCGAGGTATTCGCACTGGATACGACAAGAAG-3'; U6, 5'-

GTCGTATCCAGTGCAGGGTCCGAGGTATTCGCACTGGATACGACAAAATATGGAAC-3'. Quantitative reverse transcriptase polymerase chain reaction (qRT-PCR) was done in a Roche LightCycle480 Real-Time PCR System using the SYBR Premix Taq $\otimes$ (Takara Bio, Otsu, Japan). For quantification of mRNA levels, the real-time PCR primers were as follows: TWIST1 forward primer, 5'- AGTCTTACGAGGAGCTGCAGACG-3'; 
TWIST1 reverse primer, 5'-AGGAAGTCGATGTACCTGGCCG-3'; GAPDH forward primer 5'GCACCGTCAAGGCTGAGAAC-3'; GAPDH reverse primer 5'-TGGTGAAGACGCCAGTGGA-3'. For quantification of miRNA levels, the forward quantitative PCR (qPCR) primers used were as follows: miR543, 5'-CGGCGGAAACATTCGCGG-3'; U6, 5'-TGCGGGTGCTCGCTTCGGCAGC-3'. The reverse qPCR primer 5'-CCAGTGCAGGGTCCGAGGT-3' was used to the above miRNA. The relative transcript level of mRNA or mature miRNA was calculated using the $2^{-\triangle \Delta C t}$ method. The transcription levels of human U6 or GAPDH were used as an endogenous control.

Stabe miRNA expression cell lines

Lentiviruses containing GFP-miR-543 or GFP-negative control miRNA vector were purchased from Genepharma, Inc (Shanghai, China). SGC7901 and AGS cells were pre-seeded in a 6-well plate overnight and infected with $100 \mu \mathrm{l}$ of virus. Infected cells were selected by adding $400 \mathrm{ng} / \mathrm{ml}$ puromycin for 5 days. Stable cell lines were verified by qRT-PCR.

Cell Proliferation, Cell Cycle Assay And Soft-agar Colony Formation

For cell proliferation assay, $2 \times 10^{3}$ cells were seeded into a 96 -well plate format. And after $24,48,72,96$, or 120 hours, cells were incubated in $10 \%$ MTS (Promega) diluted in culture medium at $37^{\circ} \mathrm{C}$ for 2 hours. And the absorbance at $492 \mathrm{~nm}$ was detected using a SpectraMax M5 multimode plate reader (Molecular Devices).

For cell cycle analysis, a total of $1 \times 10^{6}$ cells were harvested, washed with PBS twice, fixed in $70 \%$ cold ethanol, incubated with staining solution $(100 \mu \mathrm{g} / \mathrm{ml}$ propidium iodide, $50 \mu \mathrm{g} / \mathrm{ml}$ RNase A, $0.1 \%$ Triton X100 in PBS) for $30 \mathrm{~min}$ at $37^{\circ} \mathrm{C}$ in the dark, and analyzed by flow cytometry.

A 1.5 -ml base layer of agar (0.6\% agar in DMEM with $10 \%$ FBS) was allowed to solidify in a six-well flatbottomed plate before the addition of $1.5 \mathrm{ml}$ of cell suspensions containing 2,000 cells in $0.3 \%$ agar in DMEM with $10 \%$ FBS. Colonies were allowed to grow for 14 days at $37{ }^{\circ} \mathrm{C}$ with $5 \% \mathrm{CO}_{2}$ before imaging.

Cell Migration, And Invasion Assays

Migration and invasion assays were performed using a Boyden chamber system with a polycarbonate membrane (8- $\mu \mathrm{m}$ pore size; 24 -well plate; Costar). For the invasion assay, the chambers were pre-coated with $50 \mu \mathrm{l} / \mathrm{cm}^{2}$ of matrigel matrix (BD Biosciences). The cells were re-seeded $\left(2 \times 10^{4}\right.$ cells) in the upper chamber containing serum-free medium. And the lower chamber contained $0.5 \mathrm{ml}$ medium supplemented with $10 \%$ FBS. After incubation, cells on the upper surface of each filter were wiped off with a cotton swab. Cells on the lower surface of membrane were fixed with $4 \%$ formaldehyde, stained with $0.1 \%$ crystal violet, washed with water and air-dried and counted under the microscope.

Transient Transfection 
A TWIST1 expression plasmid (pEZ-M13-TWIST1, catalog No.: EX-U1219-M13) and a control vector were purchased from GeneCopoeia, Inc (Guangzhou, China). miRNA-543 inhibitor and negative control were purchased from GenePharma, Inc (Shanghai China). Transfection of plasmids or siRNAs was performed using the Lipofectamine 2000 reagent (Invitrogen) according to the manufacturer's instructions.

TWIST1 Gene 3'UTR Luciferase Reporter Assay

The TWIST1 3' UTR was PCR amplified from SGC7901 cDNA and cloned into the Sacl/Nhe I site of the pGL3 luciferase reporter plasmid (Promega). The primers used were: TWIST1-3' UTR- forward, GGCGAGCTCGGAGACCTAGATGTCATTGTT; TWIST1-3' UTR- reverse, CTAGCTAGCCCCTCAGAGGAAGGATGAA. 293T cells were plated at a density of $5 \times 10^{4}$ cells per well in 24-well plate format and allowed to settle for 24 hours. Cells were transfected with pRL-TK renilla plasmid (15 ng/well), pGL-3 luciferase reporter plasmids (150 ng/well), and miR-543 mimic ( $50 \mathrm{nmol} / \mathrm{L}$ ) or scramble (50 nmol/L) using the Lipofectamine 2000 reagent (Invitrogen). Luciferase and renilla signals were determined 48 hours after transfection using a Dual Luciferase Reporter Assay Kit (Promega).

Xenograft Tumor Model

Animal tests were approved by the National Institutes of Health Guide for the Care and Use of Laboratory Animals and approved by the Animal Care and Use Committee of Sun Yat-Sen University. Female BALB/c $\mathrm{(nu} / \mathrm{nu})$ mice 4- to 6-week old were purchased from the animal facility center of Sun Yat-Sen University (Guangzhou, China). SGC7901 cells $\left(3 \times 10^{6}\right.$ cells) transfected with either the vector overexpressing miR543 or the negative control vector were inoculated subcutaneously into the right anterior flank of nude mice. Five mice were used in each group. The volume of the implanted tumor was measured at every 3 days with a vernier caliper, using the formula: $V=L \times W^{2} \times \pi / 6$; where $L$ is the length, $W$ is the width and $V$ is given in $\mathrm{mm}^{3}$. The mice were sacrificed and the tumors were weighed 3 weeks after inoculation.

Immunoblot Analysis And Immunohistochemistry

Immunoblot analysis was performed as previously described [25] using anti-TWIST1 antibody (1:1000 dilution; Abcam) and anti-GAPDH antibody (1:1000 dilution; Cell Signal Technology).

For the immunohistochemistry $(\mathrm{IHC})$ analysis, the excised tumors were fixed with $4 \%$ paraformaldehyde and sectioned into $6-\mu \mathrm{m}$ sections. Briefly, after deparaffinization, antigen retrieval and quenching of endogenous peroxidase activity, the slides were incubated with the primary antibody (TWIST1, 1:200 dilution, Abcam) overnight at $4{ }^{\circ} \mathrm{C}$ in a humid chamber. A negative control was obtained by replacing the primary antibody with a normal IgG. Thereafter, slides were incubated with the secondary antibody conjugated to horseradish peroxidase (HRP). Antibody binding was visualized using the DAB + Substrate Chromogen System (DAKO). Finally, sections were counterstained with hematoxylin, dehydrated, cleared, and photographed. 
The immunohistochemistry scoring was performed by two independent observers. Unequivocal nuclear staining pattern for TWIST1 was interpreted based on the intensity as negative, weak, moderate and strong. Moderate to strong nuclear staining was considered to be positive reaction. The distribution of TWIST 1 was scored as follows: negative (less than $50 \%$ of the cells being positive) and positive (where more than $50 \%$ of the cells were positive).

Statistics

Statistical analyses were performed utilizing the SPSS 13.0 software. Results are expressed as the mean \pm SD (standard deviation). Student's t-test (two-tailed) was used. A difference was considered significant if the $P$ value was less than 0.05 .

\section{Results}

TWIST1 level is upregulated in GC and associated with patient prognoses

We investigated the status of TWIST1 expression in GC tissue microarrays by immunohistochemical staining. Among our 107 cases of GC samples, 74 (69.2\%) cases showed moderate to strong nuclear staining of TWIST1 in most of the tumor cells (Fig. 1a). We further analyzed the relationship between TWIST1 expression and clinical characteristics of the patients (Table 1). Expression of TWIST1 was highly correlated with T classification, lymph node metastasis and clinical stage $(p<0.05)$. However, there was no significant correlation between the expression level of TWIST1 and gender, age, tumor differentiation, and distant metastasis. Overall survival analysis among 87 patients, which had the complete follow-up data, showed that patients in TWIST1-positive group had a poorer prognosis than those in TWIST1-negative group (Fig. 1b). 
Table 1

Correlation between Patient's Clinicopathologic Features and the Expression of TWIST1

\begin{tabular}{|c|c|c|c|c|}
\hline \multirow[t]{2}{*}{ Characteristics } & \multirow[t]{2}{*}{$\mathrm{n}$} & \multicolumn{2}{|l|}{ TWIST1 } & \multirow[t]{2}{*}{ p-value } \\
\hline & & - & + & \\
\hline \multicolumn{5}{|l|}{ Gender } \\
\hline Male & 66 & $19(28.8 \%)$ & $47(71.2 \%)$ & 0.560 \\
\hline Female & 41 & $14(34.1 \%)$ & $27(65.9 \%)$ & \\
\hline \multicolumn{5}{|l|}{ Age (years) } \\
\hline$<60$ & 64 & $17(26.6 \%)$ & $47(73.4 \%)$ & 0.242 \\
\hline$\geq 60$ & 43 & $16(37.2 \%)$ & $28(62.8 \%)$ & \\
\hline \multicolumn{5}{|l|}{ Tumor type } \\
\hline Intestinal & 45 & 15(33.3\%) & $30(66.7 \%)$ & 0.634 \\
\hline Diffuse/Mix & 62 & $18(29.0 \%)$ & $44(71.0 \%)$ & \\
\hline \multicolumn{5}{|l|}{ T classification } \\
\hline $\mathrm{T} 1 / 2$ & 27 & $14(51.9 \%)$ & $13(48.1 \%)$ & 0.006 \\
\hline T3/4 & 80 & 19(23.8\%) & $61(76.2 \%)$ & \\
\hline \multicolumn{5}{|l|}{$\begin{array}{l}\text { Lymph node } \\
\text { metastasis }\end{array}$} \\
\hline Positive & 82 & $19(23.2 \%)$ & 63(76.8\%) & 0.002 \\
\hline Negative & 25 & $14(56.0 \%)$ & $11(44.0 \%)$ & \\
\hline \multicolumn{5}{|l|}{$\begin{array}{l}\text { Distant } \\
\text { metastasis }\end{array}$} \\
\hline Positive & 11 & $1(18.2 \%)$ & $10(81.8 \%)$ & 0.099 \\
\hline Negative & 96 & $32(36.5 \%)$ & $64(63.5 \%)$ & \\
\hline \multicolumn{5}{|l|}{ Clinical stage } \\
\hline $\mathbb{\nabla / \mathbb { Z }}$ & 59 & $24(40.7 \%)$ & $35(59.3 \%)$ & 0.015 \\
\hline $\mathbb{\nabla} / \mathbb{\Delta}$ & 48 & $9(18.8 \%)$ & $39(81.3 \%)$ & \\
\hline
\end{tabular}

MiR-543 Inhibits TWIST1 Expression In Gastric Cancer 
Consistence with our GC tissue data, we also found that the protein level of TWIST1 was upregulated in gastric cancer cell lines compared to gastric epithelial cell line (Fig. 2a). Interestingly, miR-543 has been shown to repress the EMT in breast cancer by targeting TWIST1 [26]. Therefore, we asked whether enforced expression of miR-543 would also affect TWIST1 expression in gastric cancer cells. To validate the effect of miR-543 on the inhibition of TWIST1 expression, AGS and SGC7901 cells stably overexpressing miR-543 were established (Fig. 2b). Overexpression of miR-543 in AGS and SGC7901 cells greatly reduced the protein level of TWIST1 (Fig. 2c). Moreover, the luciferase activity of miR-543transducted cells was significantly decreased compared with the control cells (Fig. 2d). Collectively, these results established TWIST1 as a target of miR-543.

Overexpression of miR-543 inhibits gastric cancer cell growth and invasiveness in vitro

Next, we investigated the effect of miR-543 on the biological behavior of GC. Cell proliferation was examined by the MTS proliferation assay. Enforced expression of miR-543 caused a significant reduction in cell proliferation compared with the control group (Fig. 3a). To assess the tumorigenic effects of miR543 in AGS and SGC7901 cells, we performed soft agar colony formation assays in cells overexpressing miR-543. As shown in Fig. 3b, enforced expression of miR-543 in AGS and SGC7901 cells caused significant inhibition of their anchorage-independent growth, as indicated by reduction in colony number and colony size on soft agar. The cell cycle analysis further confirmed this observation, indicating that overexpression of miR-543 induced cell cycle arrest in G0/1 phase and a reduction of the S-phase cell population (Fig. 3c). To study the cell apoptosis, the cells was stained with Annexin V and PI. No significant change of apoptosis was detected between miR-543 overexpressing cells and control cells (Supplementary Fig. S1).

To further detect whether miR-543 is associated with the progression of gastric cancer, we analyzed the effect of miR-543 expression on the migratory and invasive behavior of AGS and SGC7901 cells. We found that introduction of miR-543 markedly reduced cell motility and invasiveness of gastric cancer cell lines in vitro (Fig. 3d, e). Collectively, these data indicate that miR-543 inhibits the proliferation, motility and invasiveness of gastric cancer cells in vitro.

Proliferation and invasiveness of GC cells were strengthened by endogenous TWIST1 overexpression in vitro

To confirm that the effects of miR-543 are mediated through TWIST1 in gastric cancer cells, we introduced TWIST1 into miR-543-overexpressing SGC7901 cells (Fig. 4a, b). Ectopic expression of miR543 in SGC7901 cells reduced the proliferation of cells compared to the control, whereas ectopic expression of TWIST1 in miR-543-overexpressing SGC7901 cells increased the proliferation of cells (Fig. 4c). And invasive ability of SGC7901 cells inhibited with miR-543 could be strengthen by overexpressing of TWIST1 (Fig. 4d). These data indicate that enforced expression of TWIST1 reverse the inhibitory effects of miR-543 on cell proliferation and invasiveness.

miR-543 Inhibits GC Cells Growth And Metastasis In Vivo 
To further evaluate the potential effect of miR-543 on gastric cancer cell growth and metastasis in vivo, we injected SGC7901-vc or SGC7901-miR-543 cells into the flank of nude mice. We found that the mean volume of miR-543 overexpressing tumors was smaller than that of the control (Fig. 5a, b). We also found that the mean wet weigh of tumors with miR-543 overexpression was lower than that of control $(1.03 \pm$ $0.27 \mathrm{~g}$ vs $0.56 \pm 0.13 \mathrm{~g}$; Fig. $5 \mathrm{c}$ ). Moreover, we also performed immunohistochemistry to detect the expression of TWIST1 in randomly selected tumors derived from SGC7901-vc or SGC7901-miR-543 cells. The SGC7901-miR-543 tumors expressed lower levels of TWIST1 than the SGC7901-vc tumors (Fig. 5d).

Liver is the most common target organs of gastric cancer metastasis. And we found that mice bearing SGC7901-miR-543 tumors displayed significantly lower rate of liver metastases compared with control group (20.0\% compared with 100\%; Fig. 5e, f). We also found that SGC7901-miR-543 xenotransplants generated an approximately 18.8 -fold decrease in the number of liver metastases than did vector control cells (Fig. $5 \mathrm{~g}$ ). Moreover, the reduced metastatic capability of miR-543 was demonstrated by the metastasis index showing 12.5 -fold reduction than the vector (Fig. 5h).

miR-543 Is Frequently Downregulated In Gastric Cancer

To assess the expression of miR-543 in gastric cancer, quantitative real-time RT-PCR was performed to detect the expression level of miR-543 expression in gastric epithelial cell line GES-1 and four gastric cancer cell lines (AGS, SGC7901, HGC-27, BGC-823). As shown in Fig. 6a, miR-543 was downregulated in gastric cancer cell lines compared to GES-1. To further assess the expression of miR-543 in gastric cancer tissue, quantitative real-time RT-PCR was performed to detect the expression level of miR-543 expression in 33 pairs of gastric cancer tissue and matched adjacent normal tissue samples. The expression of miR543 was downregulated in about $75.8 \%$ of tumors ( 25 of 33 patients) compared with their adjacent normal tissues (Fig. 6b, c). Thus, these data suggest that miR-543 is frequently down-regulated in gastric cancer.

\section{Discussion}

In this study, we showed that miR-543 is downregulated in gastric cancer tissues and gastric cancer cell lines. The ectopic expression of miR-543 in gastric cancer cells was shown to inhibit cell proliferation, invasion and metastasis in vitro and in vivo by targeting TWIST1.

TWIST1 is overexpressed in many types of tumors, such as cancers from breast, liver, prostate and pancreas $[8,17-19]$. In our present study, results from statistical analysis of clinical specimens revealed that TWIST1 was clinically relevant to GC aggressiveness. Dysregulation of TWIST1 alters cell proliferation, apoptosis and correlate with the invasive and metastatic phenotype of gastric cancer [27, 28]. Knockdown of TWIST1 in gastric cancer cells leads to the inhibition of tumor growth and metastasis in xenograft tumor model [29]. Thus, reducing TWIST1 expression may inhibit the progression of GC. Being able to regulate target genes, miRNAs can potentially modulate cancer development and progression [30]. It was recently reported that TWIST1 is directly regulated by miR- 543 in breast cancer cells [26]. Consistently, our data showed that ectopic expression of miR-543 reduced the expression of 
TWIST1 in GC cell lines. The result of luciferase array indicates that miR-543 directly target TWIST1 by binding TWIST1 3'UTR. Moreover, we detected the expression of TWIST1 in tumor tissue sections collected from SGC7901-vc or SGC7901-miR-543 cells and found that the miR-543-overexpressing tumors expressed lower levels of TWIST1 than the control group. These data indicate that TWIST1 expression can be regulated by miR-543 in GC.

MiR-543 has been found to be deregulated in some malignancies, including glioblastoma and breast carcinoma $[26,31]$. It has been shown that aberrant expression of miR-543 contributes to the malignant phenotypes of cancer cells in vitro, such as proliferation, migration and invasion [32,33]. On the other hand, miR-543 functions as an oncogene in hepatocellular carcinoma [34]. Thus, miR-543 may play different roles in different cancers. Here, we found that miR-543 was under-expressed in gastric cancer tissue samples and gastric cancer cell lines. And enforcing miR-543 was shown to inhibit gastric cancer cell proliferation in vitro. Besides, anchorage-independent growth is one of the hallmarks of malignant transformed cells, and critical for oncogenesis of tumor cells. Our soft agar colony formation results indicated that ectopic miR-543 can reverse the malignant character of gastric cancer cells. In vivo xenograft tumor model further confirms that miR-543 can repress the gastric cancer growth. Transwell migration and Matrigel invasion assays also demonstrated that miR- 543 significantly inhibits the motility and invasiveness of gastric cancer. Furthermore, we found that miR-543 significantly reduces liver metastases of gastric cancer cells in vivo mouse models. Taken together, our study indicates that miR543 play a very important role in gastric cancer development and metastases.

\section{Conclusion}

In conclusion, we have shown that miR-543 is downregulated in GC, and miR-543 can suppress tumor growth and metastasis in GC in vitro and in vivo by targeting TWIST1. The newly identified miR$543 /$ TWIST1 pathway provides novel insight into the molecular regulating progression and metastasis in GC. Our study indicates the intriguing possibility of using miR-543 as a promising therapeutic target for gastric cancer.

\section{Abbreviations}

GC: Gastric cancer; EMT: Epithelial-mesenchymal transition; GAPDH: Glyceraldehyde-3-phosphate dehydrogenase; TMA: Tissue microarray; IHC: Immunohistochemistry; qRT-PCR: Quantitative reverse transcriptase polymerase chain reaction; qPCR: Quantitative PCR.

\section{Declarations}

\section{Ethics approval and consent to participate}

The current study was conducted with the approval of the Ethics Committee of Sun Yat-Sen Memorial Hospital. Written informed consent documentations were obtained from all participants prior to the 
experiment. All animal experiments were performed in strict accordance with the principles and procedures of Guide for the Care and Use of Laboratory Animal by the National Institutes of Health.

\section{Consent for publication}

All presentations of case reports have consent for publication.

\section{Availability of data and materials}

The datasets used and/or analyzed during the current study are available from the corresponding author on reasonable request.

\section{Competing interests}

The authors declare no competing interests.

\section{Funding}

This work was supported by grants from Medical Scientific Research Foundation of Guangdong Province (A2016210, A2018012), National Natural Science Foundation of China (Grant No. 81972741, 81874057, 81672408, 81572396, 81302140), Medical Science Foundation of Sun Yat-sen University (19ykpy109), Natural Science Foundation of Guangdong Province (2018A030310227), Guangdong Science and Technology Department (2017B030314026).

\section{Author' contributions}

YTC, $\mathrm{KHH}$, and HML conceived and designed the research work. GDL, XNL, and SJC performed the experiments and analyzed the data. GDL, JJL, YQL, SXC, and XGL co-wrote the paper. All authors read and approved the final manuscript.

\section{Acknowledgements}

None.

\section{Author details}

${ }^{1}$ Department of Gastroenterology, Sun Yat-Sen Memorial Hospital, Sun Yat-Sen University, Guangzhou 510120, China. ${ }^{2}$ Guangdong Provincial Key Laboratory of Malignant Tumor Epigenetics and Gene Regulation, Medical Research Center, Sun Yat-Sen Memorial Hospital, Sun Yat-Sen University, Guangzhou 510120, China. ${ }^{3}$ Department of Oncology, Sun Yat-Sen Memorial Hospital, Sun Yat-Sen University, Guangzhou 510120, China. ${ }^{4}$ Department of Billary and Pancreatic Surgery, Sun Yat-Sen Memorial Hospital, Sun Yat-Sen University, Guangzhou 510120, China.

\section{References}


1. Shen L, Shan YS, Hu HM, Price TJ, Sirohi B, Yeh KH, et al. Management of gastric cancer in Asia: resource-stratified guidelines. Lancet Oncol. 2013;14(12):e535-47.

2. Siegel RL, Miller KD, Jemal A. Cancer statistics, 2018. CA. CA Cancer J Clin. 2018;68(1):7-30.

3. Karimi P, Islami F, Anandasabapathy S, Freedman ND, Kamangar F. Gastric cancer: descriptive epidemiology, risk factors, screening, and prevention. Cancer Epidemiol Biomarkers Prev. 2014;23(5):700-13.

4. Yu J, Huang C, Sun Y, Su X, Cao H, Hu J, et al. Effect of Laparoscopic vs Open Distal Gastrectomy on 3-Year Disease-Free Survival in Patients With Locally Advanced Gastric Cancer: The CLASS-01 Randomized Clinical Trial. JAMA. 2019;321(20):1983-1992.

5. Thiery JP, Acloque H, Huang RY, Nieto MA. Epithelial-mesenchymal transitions in development and disease. Cell. 2009;139(5):871-90.

6. Jiang J, Wang K, Chen Y, Chen H, Nice EC, Huang C. Redox Regulation in Tumor Cell EpithelialMesenchymal Transition: Molecular Basis and Therapeutic Strategy. Signal Transduct Target Ther. 2017;2:17036.

7. Rosivatz E, Becker I, Specht K, Fricke E, Luber B, Busch R, et al. Differential expression of the epithelial-mesenchymal transition regulators snail, SIP1, and twist in gastric cancer. Am J Pathol. 2002;161(5):1881-91.

8. Castanon I, Baylies MK. A Twist in fate: evolutionary comparison of Twist structure and function. Gene. 2002;287(1-2):11-22.

9. Yang J, Mani SA, Donaher JL, Ramaswamy S, Itzykson RA, Come C, et al. Twist, a master regulator of morphogenesis, plays an essential role in tumor metastasis. Cell. 2004;117(7):927-39.

10. Ansieau S, Bastid J, Doreau A, Morel AP, Bouchet BP, Thomas C, et al. Induction of EMT by twist proteins as a collateral effect of tumor-promoting inactivation of premature senescence. Cancer Cell. 2008;14(1):79-89.

11. Morel AP, Hinkal GW, Thomas C, Fauvet F, Courtois-Cox S, Wierinckx A, et al. EMT inducers catalyze malignant transformation of mammary epithelial cells and drive tumorigenesis towards claudin-low tumors in transgenic mice. PLoS Genet. 2012;8(5):e1002723.

12. Qian J, Luo Y, Gu X, Zhan W, Wang X. Twist1 promotes gastric cancer cell proliferation through upregulation of FoxM1. PLoS One. 2013;8(10):e77625.

13. Lee TK, Poon RT, Yuen AP, Ling MT, Kwok WK, Wang XH, et al. Twist overexpression correlates with hepatocellular carcinoma metastasis through induction of epithelial-mesenchymal transition. Clin Cancer Res. 2006;12(18):5369-76.

14. Satoh K, Hamada S, Kimura K, Kanno A, Hirota M, Umino J, et al. Up-regulation of MSX2 enhances the malignant phenotype and is associated with twist 1 expression in human pancreatic cancer cells. Am J Pathol. 2008;172(4):926-39.

15. Soini Y, Tuhkanen H, Sironen R, Virtanen I, Kataja V, Auvinen P, et al. Transcription factors zeb1, twist and snai1 in breast carcinoma. BMC Cancer. 2011;11:73. 
16. Cheng GZ, Zhang WZ, Sun M, Wang Q, Coppola D, Mansour M, et al. Twist is transcriptionally induced by activation of STAT3 and mediates STAT3 oncogenic function. J Biol Chem. 2008;283(21):14665-73.

17. Li CW, Xia W, Huo L, Lim SO, Wu Y, Hsu JL, et al. Epithelial-mesenchymal transition induced by TNF-a requires NF-KB-mediated transcriptional upregulation of Twist1. Cancer Res. 2012;72(5):1290-300.

18. Hong J, Zhou J, Fu J, He T, Qin J, Wang L, et al. Phosphorylation of serine 68 of Twist1 by MAPKs stabilizes Twist1 protein and promotes breast cancer cell invasiveness. Cancer Res. 2011;71(11):3980-90.

19. Li CW, Xia W, Lim SO, Hsu JL, Huo L, Wu Y, et al. AKT1 Inhibits Epithelial-to-Mesenchymal Transition in Breast Cancer through Phosphorylation-Dependent Twist1 Degradation. Cancer Res. 2016;76(6):1451-62.

20. Bartel DP. MicroRNAs: genomics, biogenesis, mechanism, and function. Cell. 2004;116(2):281-97.

21. Bagga S, Bracht J, Hunter S, Massirer K, Holtz J, Eachus R, et al. Regulation by let-7 and lin-4 miRNAs results in target mRNA degradation. Cell. 2005;122(4):553-63.

22. Li LZ, Zhang CZ, Liu LL, Yi C, Lu SX, Zhou X, et al. miR-720 inhibits tumor invasion and migration in breast cancer by targeting TWIST1. Carcinogenesis. 2014;35(2):469-78.

23. Zhu X, Shen H, Yin X, Long L, Xie C, Liu Y, et al. miR-186 regulation of Twist1 and ovarian cancer sensitivity to cisplatin. Oncogene. 2016;35(3):323-32.

24. Chen Y, Lian G, Ou G, Yang K, Chen J, Li H, et al. Inverse association between Bmi-1 and RKIP affecting clinical outcome of gastric cancer and revealing the potential molecular mechanisms underlying tumor metastasis and chemotherapy resistance. Gastric Cancer. 2016;19(2):392-402.

25. Yang $\mathrm{K}$, Li Y, Lian G, Lin H, Shang $\mathrm{C}$, Zeng L, et al. KRAS promotes tumor metastasis and chemoresistance by repressing RKIP via the MAPK-ERK pathway in pancreatic cancer. Int $\mathrm{J}$ Cancer. 2018;142(11):2323-2334.

26. Haga CL, Phinney DG. MicroRNAs in the imprinted DLK1-DIO3 region repress the epithelial-tomesenchymal transition by targeting the TWIST1 protein signaling network. J Biol Chem. 2012;287(51):42695-707.

27. Cheng GZ, Zhang WZ, Sun M, Wang Q, Coppola D, Mansour M, et al. Twist is transcriptionally induced by activation of STAT3 and mediates STAT3 oncogenic function. J Biol Chem. 2008;283(21):14665-73.

28. Feng MY, Wang K, Song HT, Yu HW, Qin Y, Shi QT, et al. Metastasis-induction and apoptosisprotection by TWIST in gastric cancer cells. Clin. Clin Exp Metastasis. 2009;26(8):1013-23.

29. Hsu KW, Hsieh RH, Huang KH, Fen-Yau Li A, Chi CW, Wang TY, et al. Activation of the Notch1/STAT3/Twist signaling axis promotes gastric cancer progression. Carcinogenesis. 2012;33(8):1459-67.

30. Peng Y, Croce CM. The role of MicroRNAs in human cancer. Signal Transduct Target Ther. 2016;1:15004. 
31. Skalsky RL, Cullen BR. Reduced expression of brain-enriched microRNAs in glioblastomas permits targeted regulation of a cell death gene. PLoS One. 2011;6(9):e24248.

32. Nairismägi ML, Füchtbauer A, Labouriau R, Bramsen JB, Füchtbauer EM. The proto-oncogene TWIST1 is regulated by microRNAs. PLoS One. 2013;8(5):e66070.

33. Bing L, Hong C, Li-Xin S, Wei G. MicroRNA-543 suppresses endometrial cancer oncogenicity via targeting FAK and TWIST1 expression. Arch Gynecol Obstet. 2014;290(3):533-41.

34. Yu L, Zhou L, Cheng Y, Sun L, Fan J, Liang J, et al. MicroRNA-543 acts as an oncogene by targeting PAQR3 in hepatocellular carcinoma. Am J Cancer Res. 2014;4(6):897-906.

\section{Figures}

a

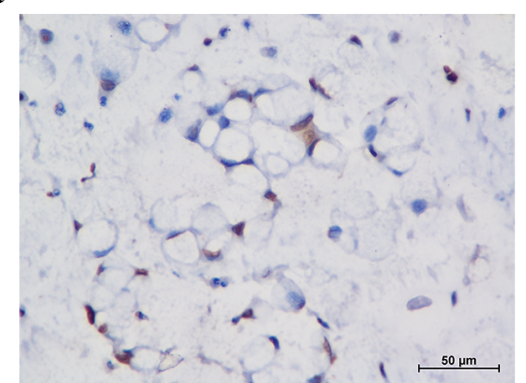

Diffuse type, TWIST1 (-)

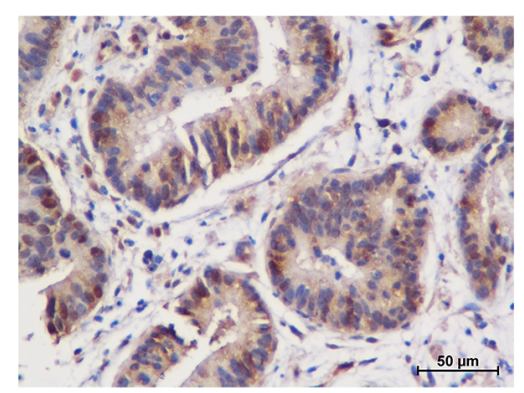

Intestinal type, TWIST1 (-)

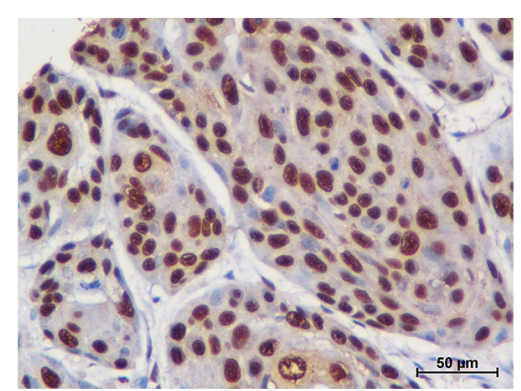

Diffuse type, TWIST1 (+)

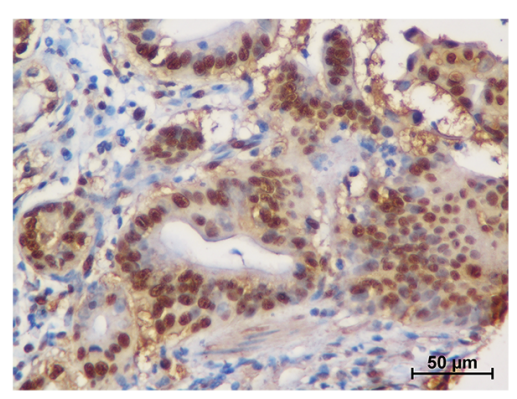

Intestinal type, TWIST1 (+) b

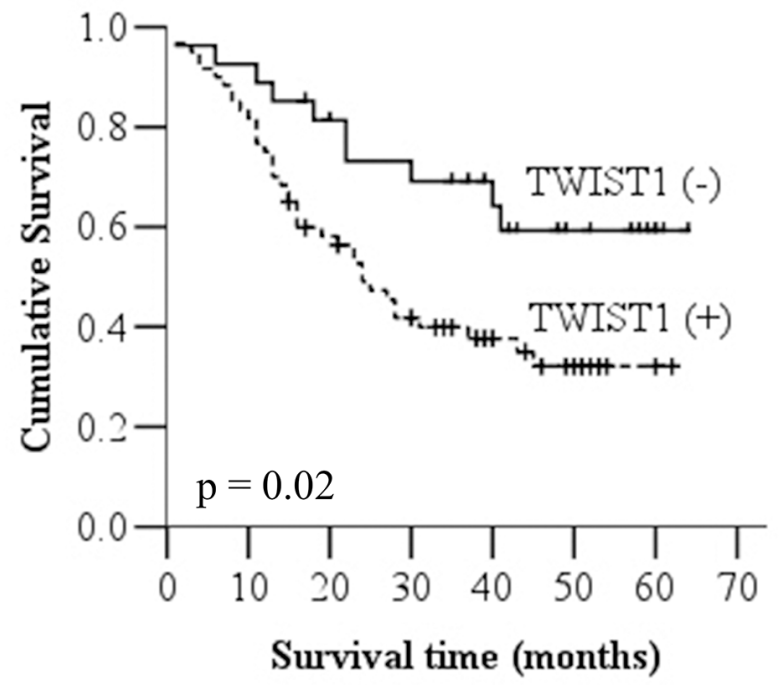

\section{Figure 1}

TWIST1 correlates with patient prognoses in gastric cancer. a Analysis of TWIST1 protein expression by immunohistochemistry in gastric cancer tissues. Representative images are shown. b Kaplan-Meier analysis of overall survival time. 
$\mathrm{a}$

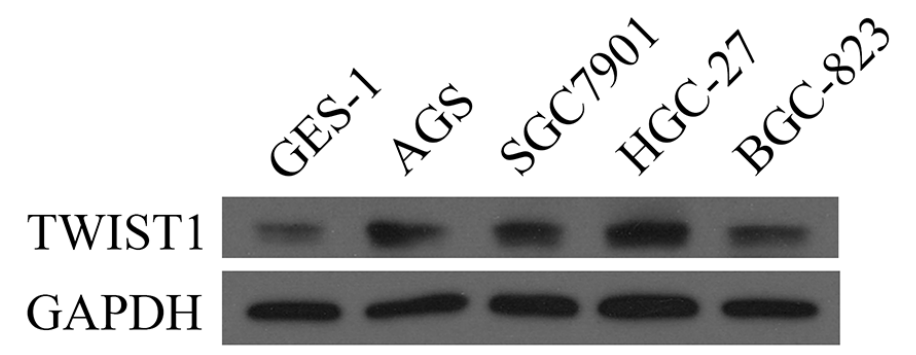

$\mathrm{b}$

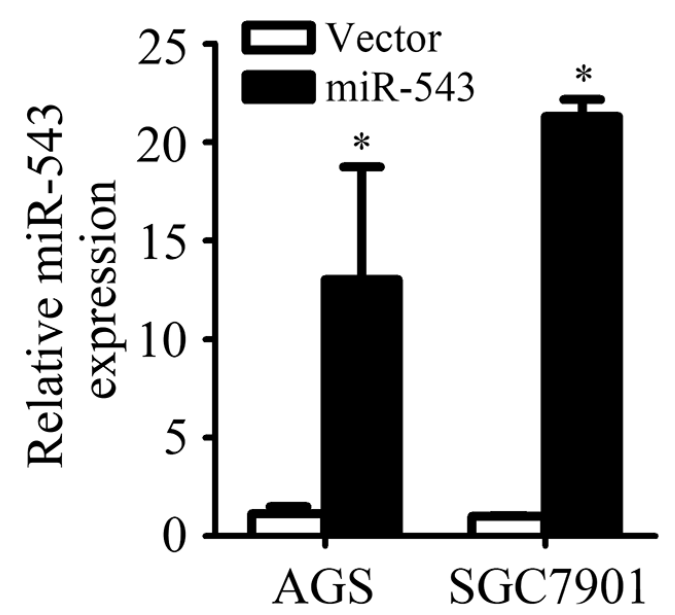

$\mathrm{C}$

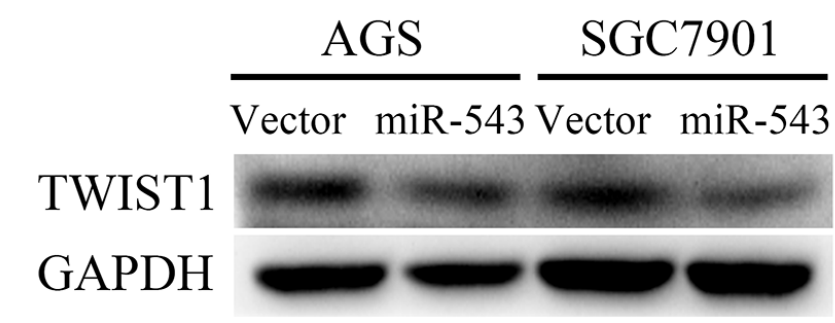

d

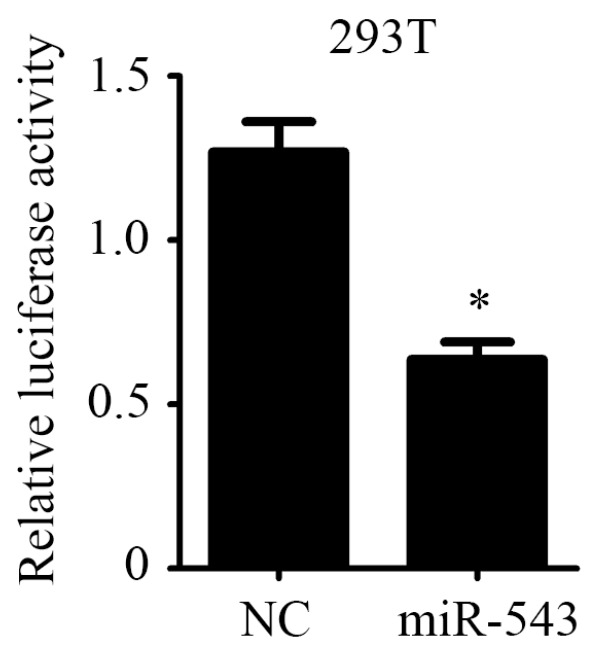

\section{Figure 2}

MiR-543 directly targets TWIST1. a Relative expression of miR-543 in four gastric cancer cell lines (AGS, SGC7901, HGC-27, BGC-823) and human nonmalignant gastric cell line (GES-1) was determined by WB analysis. b The stably up-regulation of miR-543 in AGS and SGC7901 cells by lentiviruses infection was verified by qRT-PCR. c WB of TWIST1 expression in vector- or miR-543-transduced cells. GAPDH was used as a loading control. $d$ Luciferase assay of cells transfected with a pGL3-TWIST1-3'UTR reporter with 50 nM of miR-543 mimic or NC. ${ }^{*}, \mathrm{P}<0.05$. 
a
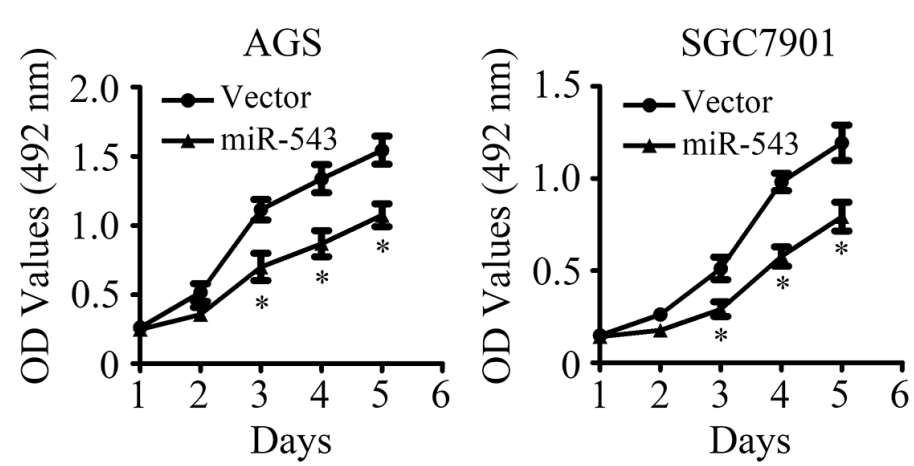

c

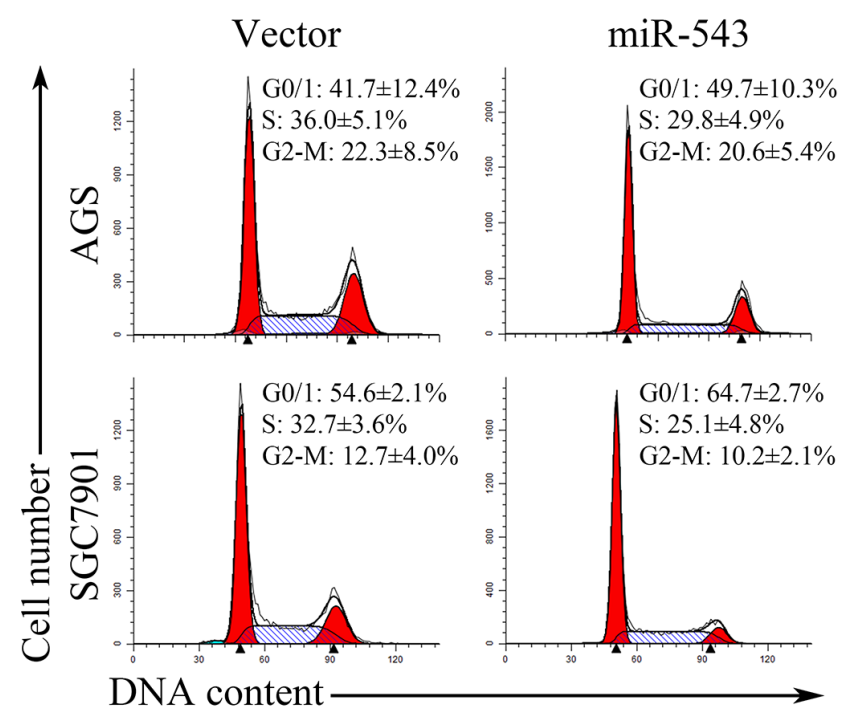

d
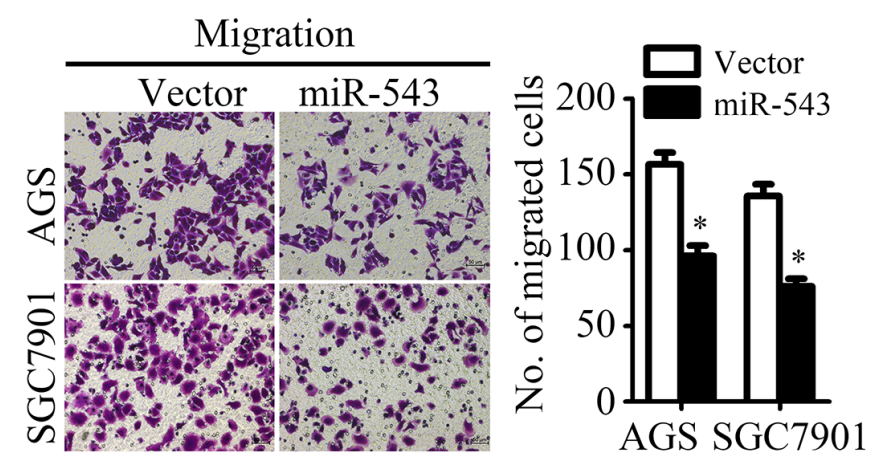

b
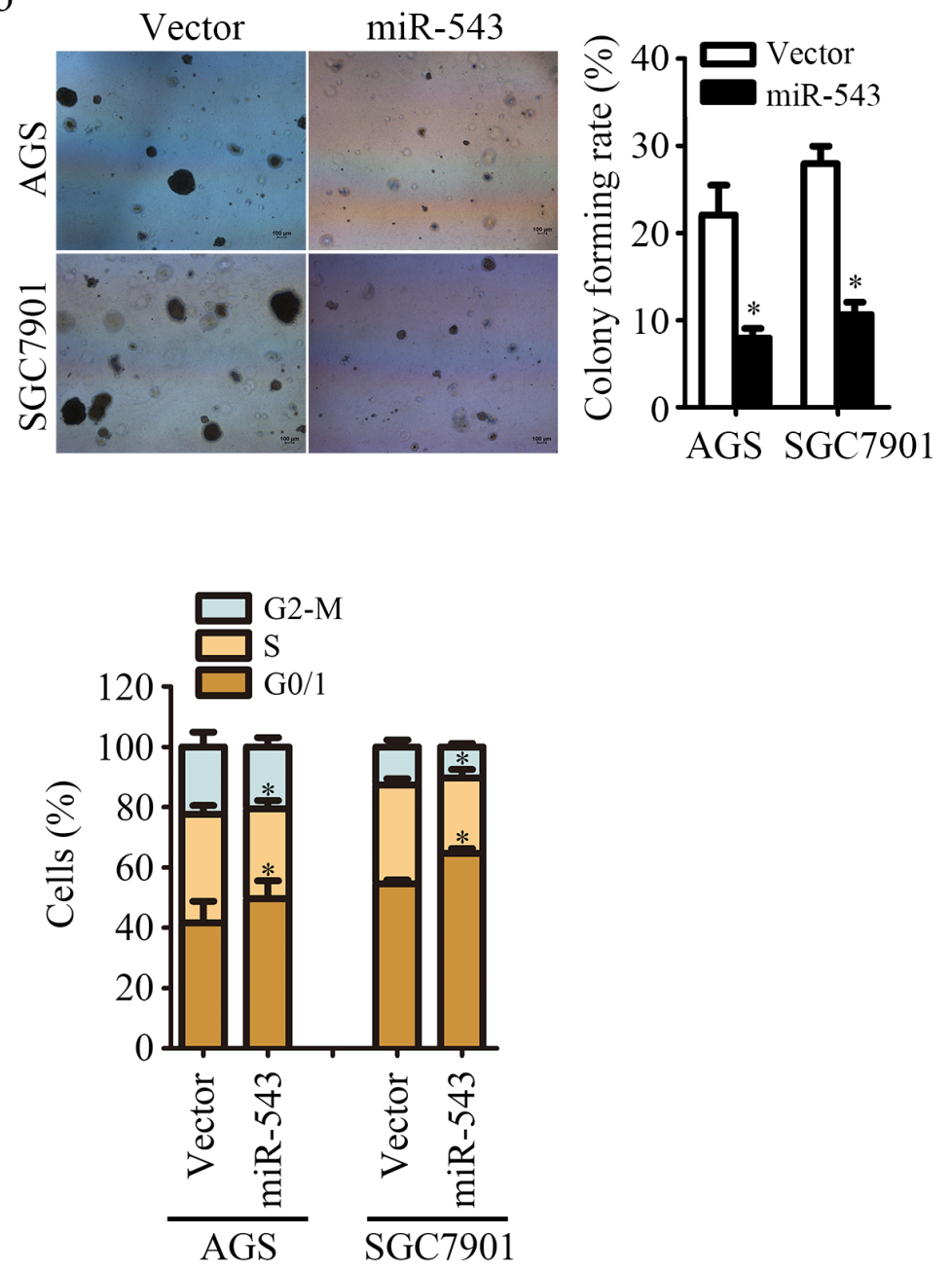

e

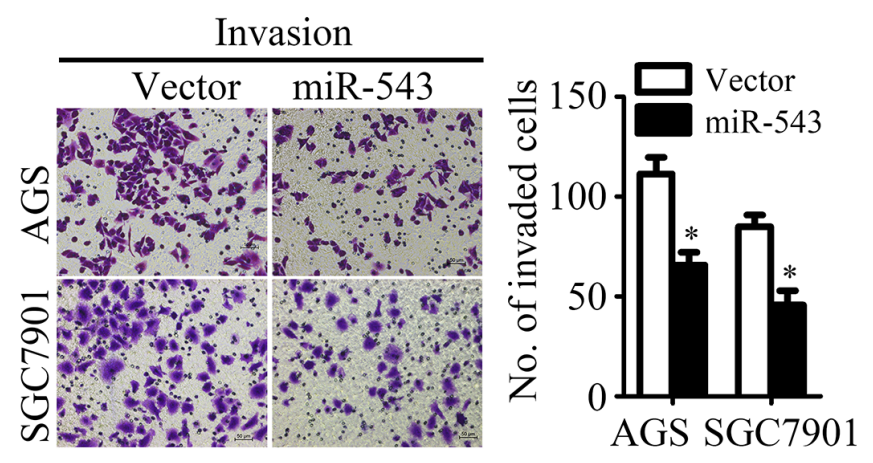

Figure 3

MiR-543 inhibits gastric cancer cell growth, migration and invasion in vitro. a The growth of indicated cells was measured by MTS assays at different time periods. b Representative micrographs and quantification of colonies verified by anchorage-independent growth assay. Magnification for identification of colony formation is $\times 40$. Scale bar: $100 \mu \mathrm{m} .{ }^{*}, \mathrm{P}<0.05$. c Effect of miR-543 on cell cycle distribution determined by flow cytometry. $d$ Transwell migration and invasion assay was performed on 
gastric cancer cells. Magnification for identification of migration and invasion is $\times 100$. Columns, mean of five fields. Scale bar: $50 \mu \mathrm{m} .{ }^{*}, \mathrm{P}<0.05$.

a

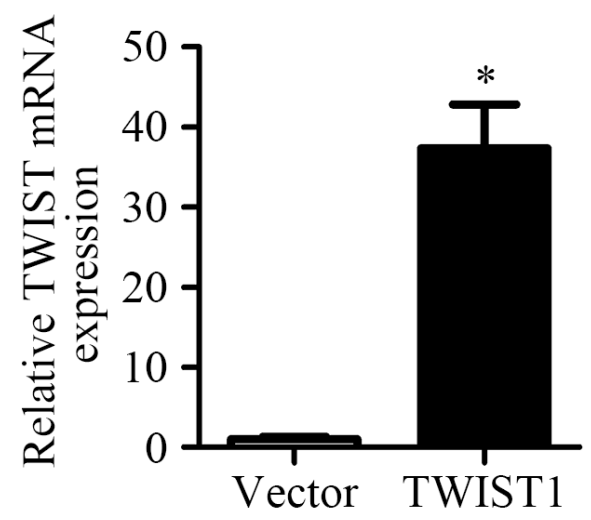

b

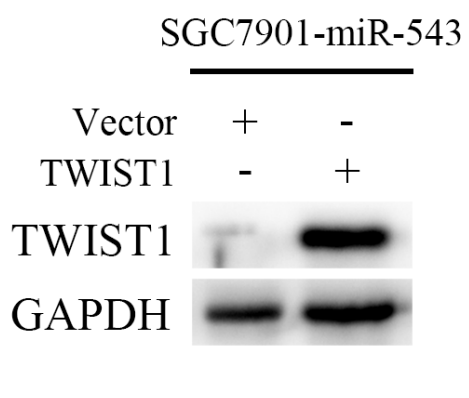

$\mathrm{c}$

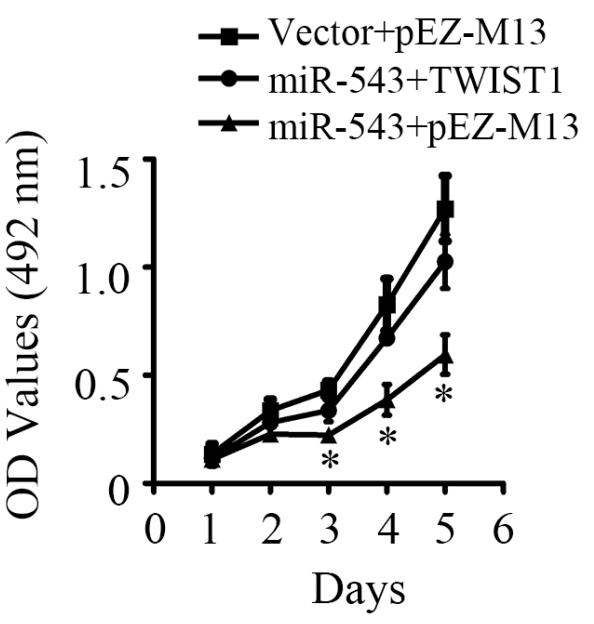

d
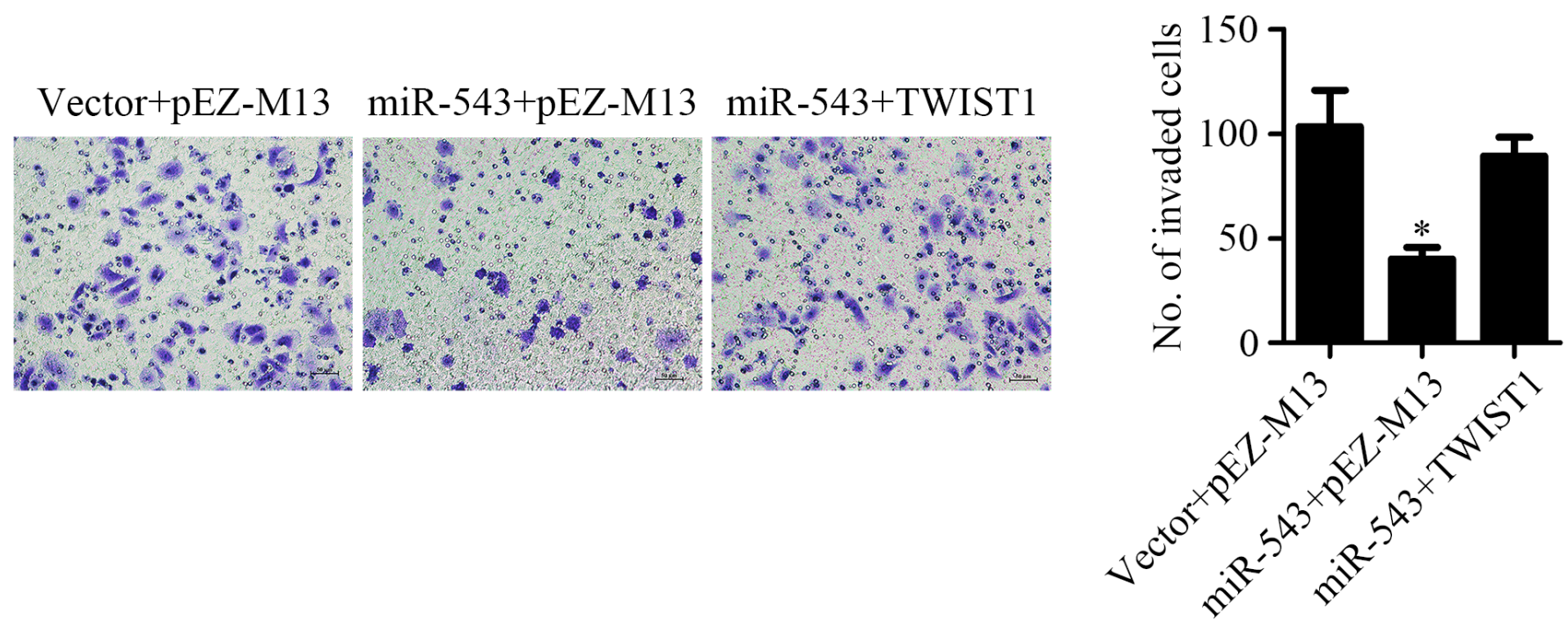

\section{Figure 4}

Enforced expression of TWIST1 reverse the inhibitory effects of miR-543 on cell proliferation and invasiveness. a The up-regulation of TWIST1 in SGC7901-miR-543 cells by transient transfection was verified by qRT-PCR. b The up-regulation of TWIST1 in SGC7901-miR-543 cells by transient transfection was verified by WB analysis. c The growth of indicated cells was measured by MTS assays at different time periods. $d$ The invasive properties of the indicated cells were evaluated by Transwell invasion assay. Magnification for identification of migration and invasion is $\times 100$. Columns, mean of five fields. Scale bar: $50 \mu \mathrm{m} .{ }^{*}, \mathrm{P}<0.05$. 
a

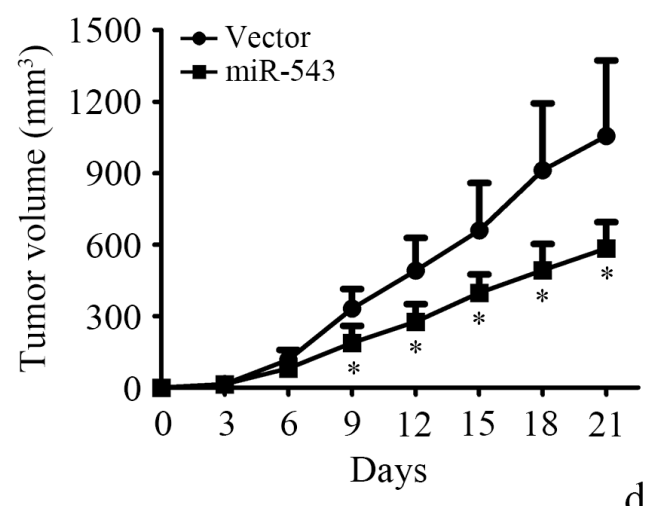

c

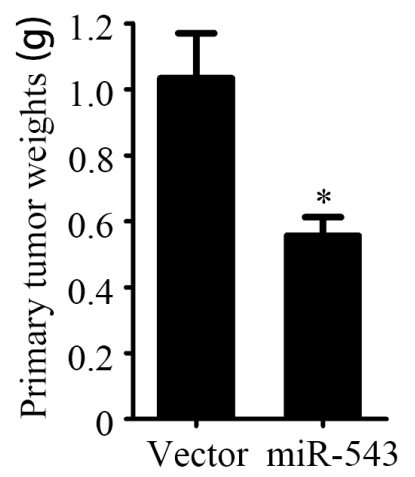

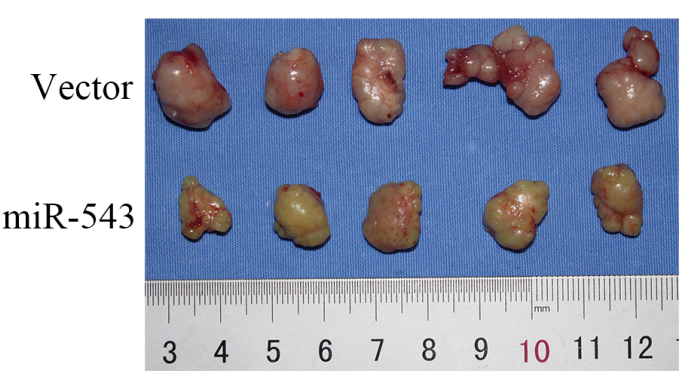

H\&E

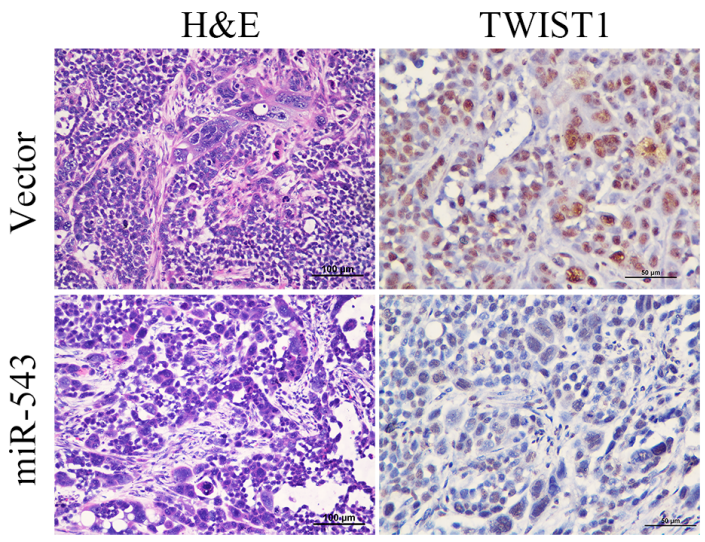

g

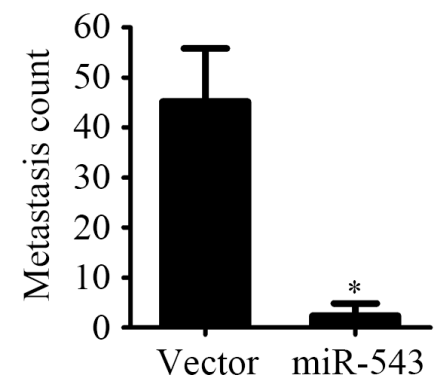

h

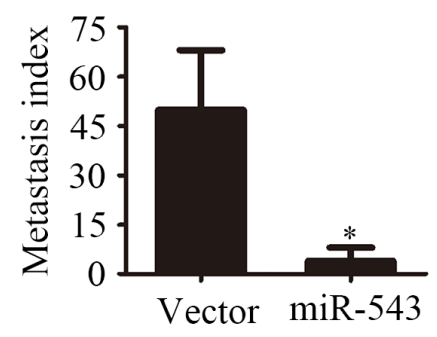

\section{Figure 5}

Overexpression of miR-543 inhibits xenograft tumor growth and metastasis of SGC7901 cells. a Tumor volumes were measured as described in Methods and plotted as the mean \pm SD. $b$ Image of the xenograft tumors. c Primary tumor weights in nude mice that received transplants of indicated cells. $d$ Expression of TWIST1 was measured by immunohistochemistry in the tissue extracted from mice receiving an orthotopic injection of indicated cells. Scale bar for H\&E staining: $100 \mu \mathrm{m}$. Scale bar for IHC staining: 50 
$\mu \mathrm{m} .{ }^{*}, \mathrm{P}<0.05$. e Representative bright-field imaging of the livers. On days 21 , mice receiving transplants of indicated cells were anesthetized and the livers were collected. $f$ Liver metastases were confirmed by H\&E staining. Scale bar: $100 \mu \mathrm{m}$. Arrows indicate the liver metastasis. g Number of visible surface metastatic lesions in livers. h Metastasis index (number of metastases per primary tumor weight) in mice receiving transplants of indicated cells. * $\mathrm{P}<0.05$.

a

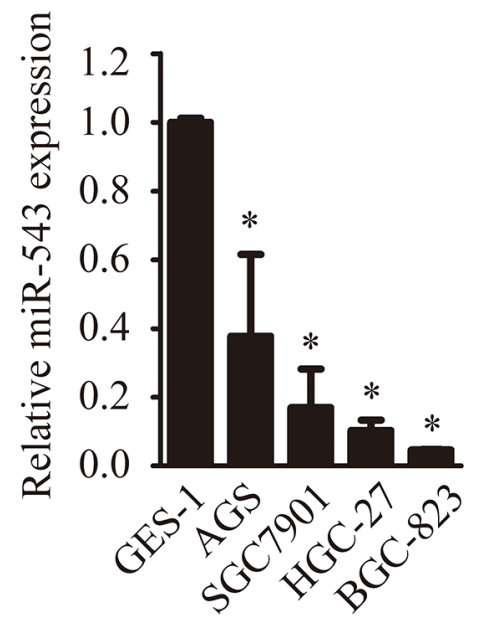

$\mathrm{b}$

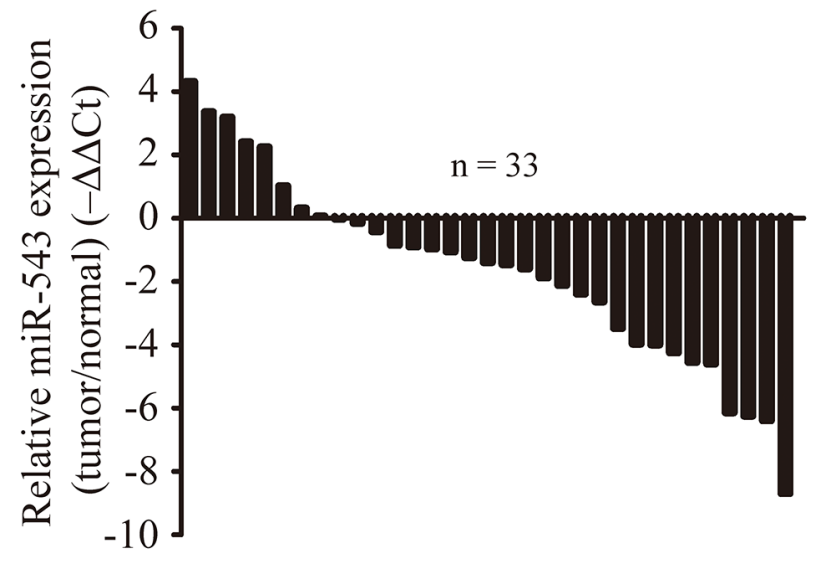

$\mathrm{c}$

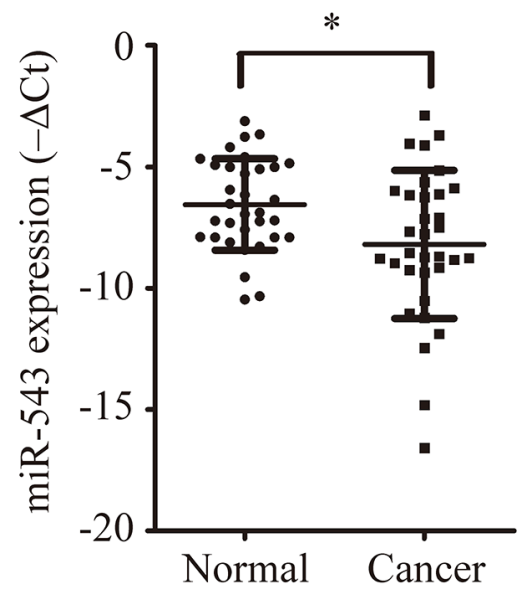

Figure 6

MiR-543 expression is frequently downregulated in human gastric cancer. a Relative expression of miR543 in four gastric cancer cell lines (AGS, SGC7901, HGC-27, BGC-823) and human nonmalignant gastric cell line (GES-1) was determined by qRT-PCR. $b$ Relative expression of miR-543 determined by qRT-PCR in 33 pairs of gastric cancer tissues compared with matching adjacent nontumor tissues. $c$ The average level of miR-543 in GC tissues and compared matching adjacent nontumor tissues. ${ }^{*}, \mathrm{P}<0.05$.

\section{Supplementary Files}

This is a list of supplementary files associated with this preprint. Click to download.

- fig.S1.tif

- fig.S2.tif

- Supplementary.doc 